\title{
THE SIGNIFICANCE OF SMALL TRACES OF BLOOD IN THE URINE.
}

\author{
By GEOFFREY E. PARKER, F.R.C.S.
}

(Surgeon to the French Hospital, London.)

In general it may be stated that the more intense the hæmaturia the easier the diagnosis is likely to prove, but the more profound will be the causative pathological changes. It is clear, therefore, that the early recognition of slight hæmaturia is of the greatest importance, because in the majority of cases the underlying pathological cause will be in its early stage and therefore more likely to be amenable to treatment. Another point to be stressed is that a small trace of blood in the urine is unlikely of itself to give rise to symptoms, unless associated with the passage of clots, and, therefore, may only be discovered in the course of a complete and careful routine investigation.

Traces of blood in the urine as an isolated physical sign also have a peculiar significance and should lead the investigator to consider the probability, whatever the age or sex of the patient, of a lesion which is apparently producing no inflammatory reaction and exerting no mechanical influence on the urinary excretory mechanism. The majority of these cases are likely to have neoplasms of the bladder, either innocent or malignant, early new growths of the renal pelvis, or of the renal parenchyma abutting fortuitously on the pelvic epithelium. It should be remembered, however, that slight hæmaturia as an isolated physical sign is not so very common when we consider the vast number of instances in which it is found in association with other symptoms, physical signs, microscopic and biochemical changes.

\section{CAUSES OF SLIGHT HÆMATURIA.}

I. Lesions confined to the genito-urinary tract.

2. Lesions of anatomically adjacent organs directly or indirectly affecting. the urinary tract.

3. Systemic disease of which hæmaturia is an isolated manifestation.

\section{Lesions confined to the Genito-urinary Tract.}

(a) Lesions of the Urethra. Urethral lesions in either sex are not common causes of slight hæmaturia. When present, the bleeding is characteristically at the beginning of micturition, clots are present and blood may be found in, or expressed from, the urethra between acts of micturition. An exacerbation of urethritis with or without a stricture, carcinoma of the male urethra at the site of a stricture, and rarely, a urethral varix may be found.

Some time ago I saw a boy, aged $2 \frac{1}{2}$ years, suffering from hæmaturia and screaming, during micturition. Circumcision had been performed with more enthusiasm than discretion and a stricture resulted at the urethral orifice with a small bleeding meatal ulcer which the child picked. at continually. Blood was quite evident microscopically and there were a few pus cells in the urine. After preliminary antiseptic treatment the usual small plastic enlarging of the meatus was done and there has been no recurrence of the hæmaturia.

Hyperamia of the verumontanum causing hæmaturia is not very uncommon and is usually associated with sexual hyper-excitability, excessive masturbation and a more or less neurotic underlying state. It is probable that the changes in 
the verumontanum are secondary. Local diathermy treatment or electro-coagulation without coincident treatment of the psychological aspect of the case may make matters worse.

Small urethral polypi and granulations, often seen round the internal urinary meatus in women, may give rise to slight hæmaturia and occasional strangury, but their importance has been exaggerated. They are certainly very common, and in the majority of cases give rise to no symptoms at all. One should not be content with such a diagnosis without a complete urological investigation to exclude other possible causes.

(b) Lesions of the Vesicles and Prostate. In men acute vesiculitis and prostatitis give rise to traces of blood in the urine from congestion of the overlying urethral mucosa, but the local acute inflammatory changes should rarely require or justify a urethroscopy for confirmation. Benign enlargement of the prostate, more particularly of its middle lobe, causes hæmaturia, but here again, if such an enlargement be found and there are no other symptoms of any interference with the mechanical outflow of urine from the bladder, this should not be considered to be the cause without a complete investigation of the urinary tract. Notes of a case are at hand of a man of 63 years, apparently in perfect health, who noticed that his urine was becoming cloudy. The prostate was moderately enlarged, but there was no trabeculation of the bladder which contained less than two ounces of residual urine and the bleeding was coming from a carcinoma of the left kidney.

(c) Lesions of the Bladder. Slight hæmaturia almost invariably accompanies acute cystitis due to the bacillus coli and less commonly occurs during congestive phases of a chronic infection, but pyuria and frequency are more evident signs. Tuberculous ulceration of the bladder is not an uncommon cause of small traces of blood in the urine. Pus cells are always present and frequency is usually intense. Calculous disease of the bladder causes an intermittent hæmaturia always in association with pyuria, unless the stone is of metabolic origin and recently descended from one or other kidney. From the third to the fifth decade the causes of hæmaturia of vesical origin are papilloma and carcinoma, and it is in these cases above all that the importance of "traces of blood" is stressed. Such tumours in their early stages offer a comparatively hopeful outlook for cure by surgical means. Of the rarer causes of vesical bleeding in small amounts, hamangiomata and varicose veins on the bladder floor should be mentioned and there is the still rarer and interesting condition of endometriosis of the bladder which produces hæmaturia during menstruation. The bleeding is caused by congestion and desquamation from the surface of the tumour which is itself produced by the migration of endometrial tissue into the bladder wall.

(d) Lesions of the Ureter. Bleeding of ureteral origin is a rare urological curiosity apart from that occasioned by the passage of small calculi. Its occurrence may be due to a papilloma or carcinoma, usually a seedling growth from the kidney, although very occasionally the tumour may be of ureteral origin. Adjacent inflammatory changes may cause bleeding from the ureter but this factor will be discussed later.

(e) Lesions of the Kidney. Hæmaturia of renal origin is not usually excessive, except in cases of injury, but a papilloma of the renal pelvis may bleed sufficiently severely and steadily to produce a profound secondary anæmia.

I recently operated on a man aged 74 who had had painless hæmaturia for four years. This had slowly increased in amount and latterly was accompanied by pus. The patient only submitted to operation on account of repeated giddy attacks induced by the profound secondary anæmia. The left kidney was pyonephrotic and contained a papilloma, a carcinoma and many calculi. 
Two unusual conditions which give rise to special difficulty in diagnosis are papillitis and hamorrhagic nephritis.

In papillitis the diagnosis may be further confused by the fact that a transient œdema of the affected pyramid may give rise to a filling defect in the pyelogram, suggesting a neoplasm. The condition usually clears up spontaneously, but I know of no method by which one can definitely exclude a neoplasm in such cases other than exploratory incision of the kidney substance and pelvis.

Hydronephrosis may also cause slight hæmaturia and is probably more common than is generally thought. In a personal series of eleven cases, hæmaturia was present in five and was the presenting symptom in three. It is due, no doubt, to transient congestion, without infection, at or near the uretero-pelvic junction.

A man of thirty-five years complained of painless hæmaturia of ten days' duration. He was apparently in perfect health and was unaware of any other abnormality. At operation the right kidney was found to be a partly-filled sac containing clear urine. Only a trace of secreting renal tissue remained in the thinned-out shell. The ureteropelvic junction was reduced to a pin hole and the ureter below this was normal, as was also the opposite kidney.

Traces of blood are commonly found immediately following a Dietl's crisis associated with renal hypermobility. In transient oxaluria, if sufficiently intense to cause renal colic, small traces of blood, sometimes not amounting to more than one or two red blood corpuscles per field, make their appearance in the urine.

Nephritis, (acute and sub-acute) cause a variable mild hæmaturia but the presence of albumin in excess and casts (which however may be scanty and late in appearing), plus the cardiac and arterial changes seen in chronic cases leads to the diagnosis. It is interesting to remember that the hæmaturia is quite frequently unilateral. A difficulty arises, commonly in elderly people, where there is associated with the nephritis a surgical lesion of the kidney.

Rarely, the first symptom of renal tuberculosis may be a mild attack of hæmaturia due to ulceration through a vessel in the wall of a small cavity, but pus cells will be present and also, as a rule, frequency of micturition and local pain. The discovery of the turbercle bacillus either in films or on culture, or on guinea pig inoculation, settles the question.

A slight hæmaturia is by no means uncommon as the initial symptom in polycystic disease, and cysts adjacent to the pelvis may produce a filling defect on pyelography suggesting a new growth. The blood urea is raised, together with the arterial tension in the later stages, but these conditions may continue for many years with the blood urea within normal limits and the kidneys not much enlarged, the left one being commonly not palpable.

A recent case of this kind came under my care in which hæmaturia was the only complaint, and the blood urea was normal. The right kidney nodular; the left kidney was not palpable while the right ascending and descending pyelograms both showed an absent upper minor calyx. The correct diagnosis was only made, therefore, on cutting down on the kidney. The peritoneum was opened and the opposite kidney was felt to be cystic too.

Very small traces of blood without infection or other abnormality of the urine when of renal origin should suggest the diagnosis of malignancy. As age advances simple growths of the renal pelvis are less common than carcinoma and simple tumours of the renal parenchyma are so rare that for practical purposes they may be ignored when considering the differential diagnosis. 


\section{Lesions of anatomically adjacent organs directly or indirectly affecting the urinary tract.}

Acute appendicitis undoubtedly may cause slight hæmaturia. The explanation is, I believe, the presence of toxic nephritis by absorption through the lymphatic and blood streams. It commonly continues for a short time after the appendix has been removed. Two personal cases exhibited this phenomenon and the appendix was not lying in any relationship to the right ureter, though no doubt this does occur.

Cases are on record of inflammatory changes in the gall-bladder and of leakage from a small perforation of a duodenal ulcer which have caused renal congestion and hæmaturia. Uterine fibroids may cause frequency and hæmaturia, especially if situated anteriorly and in relation to the bladder base, and quite irrespective of their size. There would seem to be no doubt from the cystoscopic appearances that this is due to mechanical interference with normal bladder movements causing spasm and congestion. Similarly, a pelvic appendix abscess or a pyosalpinx will produce sufficient congestion of the pelvic portion of the ureter and bladder to produce mild hæmaturia.

A localised mass associated with a perforated diverticulum of the pelvic colon was the cause of mild hæmaturia in a man of fifty. On cystoscopy an area of intense congestion was observed near the vault of the bladder on its posterior aspect, well above the left ureter and no doubt such a case, if untreated, would have led to a vesico-intestinal fistula.

Rectal and cervical growths in the course of direct spread, but before the malignant cells have invaded the lower urinary tract, will cause painful vesical spasms and hæmaturia by involvement of the hypogastric ganglia. Advanced cases with associated ulceration cause secondary inflammatory changes in the? pelvic fascial planes and no doubt this is a further contributory cause.

\section{Systemic Disease, of which Hæmaturia is an Isolated Manifestation.}

Hæmaturia may occur in the course of almost any disease which has among its characteristics a focal infection of whatever nature, associated with wide fluctuations in the temperature. The significance of the symptom, however, may vary considerably and must be assessed in conjunction with the other physical signs present. A staphylococcal septicamia, for example, associated with a large carbuncle or an endosteal abscess, may cause mild hæmaturia without urinary symptoms and usually without significance. The blood disappears if, and when, the disease subsides. A true staphylococcal carbuncle of the kidney rarely, if ever, starts in this way. On the other hand, the sudden appearance of blood in the urine of a patient suffering from mitral disease may be the first indication of malignant endocarditis.

Just as the anterior-septal artery and the capsular branches of the middle cerebral artery may give way in the hypertensive so may glomerular branches of the renal artery. The bleeding, perhaps slight at first, may rapidly become intense and even alarming. The importance of this symptom lies, of course, in the early appreciation of its cause, so that active measures may be undertaken to reduce the arterial tension.

Hamorrhagic purpura is another rare systemic cause of hæmaturia. The bleeding may be quite severe and commonly comes from one or other kidney. Help is gained in arriving at a diagnosis if, in the absence of other hæmorrhage, on cystoscopic examination small sub-epithelial petechia are seen on the bladder 
wall. There is a curious form of purpura in which the repeated hæmorrhages are always confined to the urinary tract, but even in these cases a careful history will usually elicit the fact that there is a tendency to excessive bruising after trivial trauma.

Hæmaturia in hamophilia is not, of course, uncommon, and has a grave significance on account of the relative inaccessibility to local treatment.

Hæmaturia is stated to occur in 15 per cent. of cases of leukamia but must be unusual as an initial symptom.

Traces of blood are frequently seen in the urine of cases of scurvy and at times the bleeding may be sufficient to produce clots and renal colic.

Hæmaturia is not unknown in typhoid fever and is stated to be of much graver significance if it occurs during the first week than in the later stages of the disease. At the time that blood is present the bacilli can always be cultured in the urine, a fact of some importance if, during the first week of the disease, the diagnosis has not been definitely established.

All the acute exanthemata may, in rare and very severe cases, initiate hæmaturia. It varies in intensity with the infection and disappears during recovery.

No account of bleeding from any part of the body is complete without mention of policythamia rubra, and urinary hæmorrhage is a comparatively frequent symptom.

In discussing the significance of this important, and sometimes elusive phenomenon, stress may at times appear to have been laid on the rarities of urinary pathology, while the everyday causes of hæmaturia, such as vesical growths and simple enlargements of the prostate, have scarcely been touched upon. The excuse must be made firstly, that the latter subjects have already been dealt with in the literature, and secondly, that the intention has been throughout to stress the importance of it being symptomatic of serious and possibly fatal disease, so that the patients may present themselves for treatment at a time when this will be at once simple and straightforward rather than hazardous and heroic.

REFERENCES.

Debenham, Brit. J. Surgery, 1933-34, vol. 21, p. 44.

Eisengrath and Rolnick, Urology, p. 904.

Marion, Traite d'Urologie, third edition, vol. 1, p. 176

Achard, Monde Medical, 1936, No. 876, p. 33.

Addison, Proceedings Royal Soc. Med. 1936, vol, 29, p. 1295

Weijelandt, Proceedings Royal Soc. Med. 1934, vol. 27, p. 1493. 